\title{
Localisation de la déformation par corrélation d'images numériques sur laine de verre crêpée
}

\author{
Sandra Bergonnier ${ }^{1,2}$, François Hild ${ }^{1, a}$ et Stéphane Roux ${ }^{2}$ \\ 1 LMT-Cachan, ENS de Cachan, CNRS-UMR 8535, Université Paris 6, 61 avenue du Président Wilson, 94235 Cachan Cedex, \\ France \\ 2 Laboratoire « Surface du Verre et Interfaces », UMR CNRS/Saint-Gobain 125, 39 quai Lucien Lefranc, \\ 93303 Aubervilliers Cedex, France
}

Reçu le 27 novembre 2003, accepté le 21 janvier 2006

\begin{abstract}
Résumé - Une approche multi-échelles de la corrélation d'images numériques est présentée. Elle permet de résoudre précisément les fluctuations du champ de déplacement. Cette technique de mesure est ensuite appliquée à des essais de compression et d'arrachement réalisés sur laine de verre crêpée. Les champs de déplacement et de déformation sont alors déterminés. Cette méthode révèle, lors de la réalisation d'essais de compression, des hétérogénéités de déformation, prémices de la localisation, bien avant le plateau de contrainte. Les essais d'arrachement permettent d'identifier l'amorçage et la propagation de fissures. La fiabilité et la résolution des champs de déplacements et de déformations sont validées en utilisant différents paramètres lors de l'analyse par corrélation.
\end{abstract}

Mots clés : Corrélation d'images / laine de verre / localisation de la déformation / analyse multi-échelles

\begin{abstract}
Strain localization using digital image correlation on crimped glass wool. A multiscale approach of digital image correlation is presented. It allows one to resolve accurately fine details of the displacement field. This technique is applied to compression and tearing tests carried out on crimped glass wool. Displacement and strain fields are determined. This method reveals strain heterogeneities and further localization in compression tests well below the stress plateau. Crack initiations and propagations are identified in tearing tests. Reliability and resolution of the displacement and strain fields are validated by using different parameters in the correlation analysis.
\end{abstract}

Key words: Image correlation / glass wool / strain localization / multiscale analysis

\section{Introduction}

Pour modéliser les propriétés mécaniques des matériaux, des lois de comportement validées par la réalisation d'essais mécaniques sont nécessaires. Lors de la mise en œuvre de ces essais, différentes techniques de mesure peuvent être utilisées, à savoir : la localisation d'émissions acoustiques [1], signature de microfissuration, applicable pour des matériaux fragiles ou quasi fragiles tels que le béton ou la roche, la technique des répliques [2] qui permet d'identifier des fissurations de surface, ou les méthodes optiques. Celles-ci, étant sans contact [3] et donc non perturbatrices, ont un large champ d'applications dans le domaine de la mécanique des matériaux et des structures [4]. Au cours d'essais

\footnotetext{
a Auteur correspondant :

Francois.Hild@lmt.ens-cachan.fr
}

mécaniques, les champs de déplacement mesurés peuvent faire apparaître, selon la taille de la région de l'image analysée, des hétérogénéités de déformation à la surface de l'éprouvette. Ces dernières représentent les prémices éventuelles d'une localisation de la déformation. L'apparition et le suivi de fissures peuvent également être analysés par ces techniques [5,6]. Les méthodes optiques fournissant une évaluation locale des champs cinématiques, il est possible de suivre les déformations locales et/ou déplacements tout au long de l'essai et donc d'identifier la localisation de la déformation dès son apparition [7].

Différentes techniques peuvent être utilisées pour déterminer les champs de déplacement et de déformation. Lorsqu'une méthode expérimentale met en œuvre des techniques optiques (i.e., photo) pour résoudre les problèmes mécaniques, on parle de photomécanique $[3,8]$. La photoélasticité et les méthodes de moiré sont deux 


\section{Nomenclature}

\begin{tabular}{|ll|}
\hline$b:$ & bruit \\
$f:$ & signal \\
$g:$ & signal décalé \\
$h:$ & intercorrélation \\
$k:$ & nombre entier \\
$P, Q:$ & nombres entiers \\
$\boldsymbol{u}:$ & vecteur déplacement \\
$u_{1}:$ & composante locale du déplacement selon \\
& la direction 1, pixel (ou m) \\
$\boldsymbol{x}:$ & vecteur position \\
$\delta:$ & décalage entre deux ZE voisines, pixel (ou m) \\
$\bar{\epsilon}:$ & déformation globale \\
$\epsilon_{11}:$ & déformation locale normale dans la direction 1 \\
$\boldsymbol{v}:$ & vecteur déplacement test \\
$\boldsymbol{v}_{0}:$ & évaluation initiale du vecteur déplacement test \\
$\boldsymbol{\delta} \boldsymbol{v}:$ & correction du vecteur déplacement test \\
$\star$ & $\quad$ opérateur d'intercorrélation \\
$\ll \cdot »:$ & variable muette \\
\hline
\end{tabular}

des premières méthodes développées depuis la première moitié du vingtième siècle. La photoélasticité [9-12] est toujours très utilisée dans l'industrie. Les méthodes de moiré sont des techniques versatiles qui fournissent des champs de déplacement aussi bien que des mesures topographiques [13-15]. Le développement des lasers, au début des années 1960, a permis l'émergence de nouvelles techniques. La première approche est l'interférométrie holographique $[16,17]$ qui peut être appliquée à un grand nombre de problèmes industriels [18]. Un sous-produit est la technique de «speckle» (ou tavelure) qui regroupe l'interférométrie de speckle [19-21] et la photographie de speckle [22-24]. Lorsque cette dernière est utilisée en lumière blanche, elle est appelée corrélation d'images numériques (CIN) [25-27]. Proche de la technique de vélocimétrie par imagerie de particules [28-30] très répandue en mécanique des fluides, son principe est basé sur l'appariement d'images en maximisant l'intercorrélation. En termes de préparation d'éprouvettes, la CIN s'adapte généralement bien à l'environnement d'un laboratoire d'essais mécaniques. On notera que des techniques de stéréocorrélation sont également en cours de développement [31,32]. Dans le cas présent, les déplacements hors plan restent faibles si bien que ces corrections sont inutiles.

La technique de CIN est facile à mettre en ouvre et représente un outil efficace dont la résolution peut être étendue bien en dessous du pixel (i.e., $10^{-2}$ pixel voire en dessous dans certains cas). Lorsque le déplacement entre deux images utilisées pour le calcul est trop important, le résultat donné par les méthodes classiques conduit à des erreurs significatives. Dans ce cas, l'utilisation d'une approche multi-échelles de la corrélation d'images est possible [33]. Elle autorise de grands déplacements entre les images tout en garantissant de très bonnes robustesse et résolution. Cette méthode, appliquée d'abord à des essais de compression uniaxiale puis à des essais d'arrachement, permet d'atteindre des niveaux de déformation élevés avec une très bonne résolution, alors que la plupart des

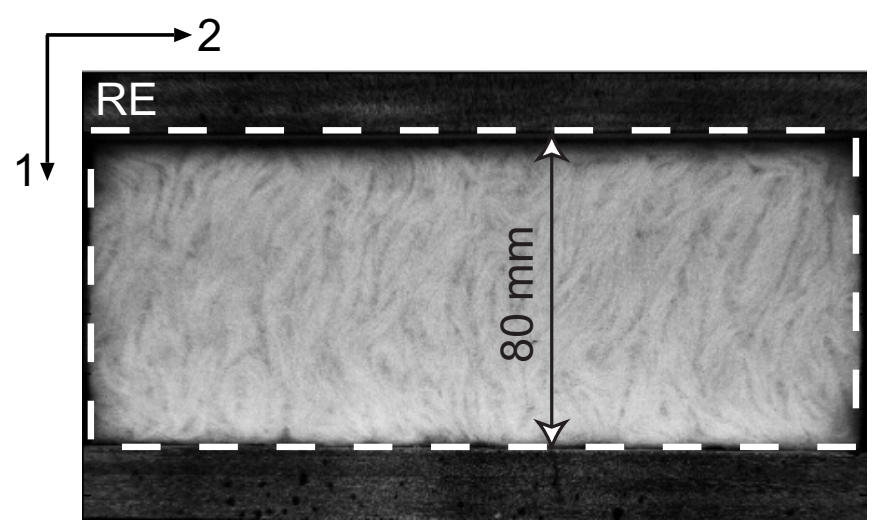

Fig. 1. Vue latérale d'un produit en laine de verre crêpée. Région d'étude (RE) pour la mesure de déplacements par corrélation d'images numériques.

autres techniques de mesures de déformation ne seraient pas applicables ou donneraient des résultats erronés.

Le but de l'étude présentée dans cet article est de montrer une application de l'approche multi-échelles de la CIN, i.e., des essais mécaniques sur laine de verre crêpée. La section 2 introduit le procédé de crêpage, opération industrielle visant à augmenter les performances mécaniques du matériau à densité donnée. La section 3 traite des deux méthodes de CIN : l'approche conventionnelle et l'approche multi-échelles. Les performances en termes d'incertitude, de résolution et de résolution spatiale sont évaluées sur un cas pratique. Enfin, les essais mécaniques réalisés sur laine de verre crêpée sont analysés à la section 4. Des essais de compression sont effectués jusqu'à - $20 \%$ de déformation globale. Des essais d'arrachement sont également réalisés sur le même type d'éprouvettes jusqu'à $+15 \%$ de déformation globale.

\section{La laine de verre crêpée}

La laine de verre est un produit constitué de fibres de verres, dont le diamètre moyen est de l'ordre de quelques micromètres et de longueur milli- ou centimétrique. Les fibres sont enchevêtrées et encollées avec une résine. De par la manière dont elle est produite, la laine a une structure stratifiée présentant des différences de densité clairement visibles sur l'épaisseur du produit. Telles quelles, les résistances à la compression et à l'arrachement de la laine sont faibles même pour des densités moyennes. Afin d'augmenter les performances mécaniques, un « crêpage » est réalisé. Cette opération consiste à effectuer une compression uniaxiale en ligne le long du plan de stratification pour imposer une rotation de l'axe d'anisotropie ou déformer les couches denses pour obtenir une texture plus isotrope (Fig. 1). Dans les deux cas, à densité donnée, de bien meilleures performances mécaniques sont obtenues. La résistance à la compression peut être augmentée d'un facteur supérieur à deux grâce au crêpage. Ceci souligne l'importance de la texture du produit pour son comportement mécanique. Ainsi une caractérisation 


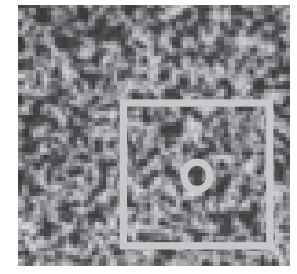

Image de référence

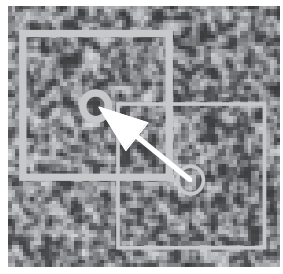

Image déformée
Fig. 2. Zone d'étude (ZE) sur les images de référence et « déformée ». Vecteur déplacement associé.

propre de la texture et de la déformation locale (et leur interdépendance) est nécessaire pour optimiser les performances du produit. Ceci motive l'étude détaillée à la section 4 .

\section{Mesure de déplacement par corrélation d'images}

La technique de CIN est particulièrement bien adaptée pour les solides «souples » pour lesquels il peut être difficile de coller des jauges de déformation qui peuvent, par ailleurs, perturber la réponse du matériau (e.g., les polymères $[34,35]$, la laine minérale $[33,36]$, le bois et le papier [37]). La CIN est basée sur l'utilisation d'un algorithme d'appariement d'images (i.e., le produit de corrélation), qui peut être effectué soit dans l'espace physique [27, 38], soit dans l'espace de Fourier [39-41]. Dans le cas présent, les calculs dans l'espace de Fourier sont utilisés. Le champ de déplacement plan est obtenu par intercorrélation d'une fenêtre d'interrogation dans l'image de la surface déformée par rapport à une image de référence. L'avantage d'une telle méthode vient du matériel nécessaire à sa mise en œuvre, c'est-à-dire une caméra CCD, une carte d'acquisition rapide, un PC et une source de lumière blanche.

Pour déterminer le champ de déplacement d'une image par rapport à une image de référence, on considère un ensemble de fenêtres d'interrogation que l'on appelle Zone d'Étude (ZE). Le but de la méthode de corrélation est d'apparier les ZE des deux images (Fig. 2). Le déplacement d'une ZE par rapport à l'autre est un décalage d'un signal bidimensionnel d'intensités numérisées par la caméra CCD. Ainsi une ZE est représentée par des fonctions $g(\boldsymbol{x})$ qui sont des perturbations d'un signal décalé $f(\boldsymbol{x}-\boldsymbol{u})$ par rapport à un signal de référence $f(\boldsymbol{x})$

$$
g(\boldsymbol{x})=f(\boldsymbol{x}-\boldsymbol{u})+b(\boldsymbol{x})
$$

où $\boldsymbol{u}$ est le vecteur déplacement plan inconnu, supposé constant localement et $b(\boldsymbol{x})$ un bruit aléatoire. Pour évaluer le déplacement $\boldsymbol{u}$, on minimise la norme de la différence entre $f(\boldsymbol{x}-\boldsymbol{v})$ et $g(\boldsymbol{x})$ par rapport à un déplacement test $v$

$$
\min _{\boldsymbol{v}}\left(\|g(.)-f(.-\boldsymbol{v})\|^{2}\right)
$$

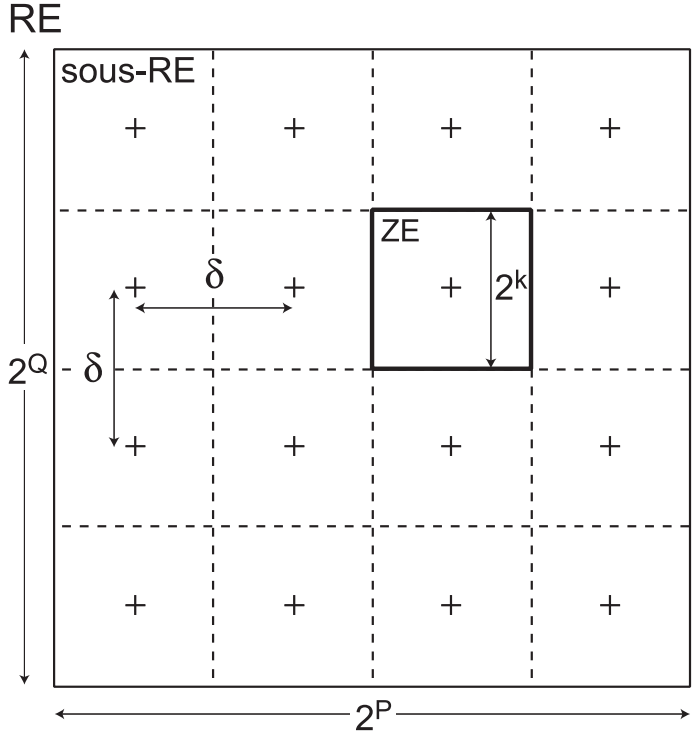

Fig. 3. Schéma de l'image de référence avec les paramètres de corrélation : taille de $\mathrm{ZE}=2^{k}$ pixels, décalage entre $\mathrm{ZE} \delta$. Dans le cas particulier de la figure on a $\delta=2^{k}$ pixels.

Lorsque l'on choisit la norme quadratique habituelle $\|f\|^{2}=\iint|f(\boldsymbol{x})|^{2} \mathrm{~d} \boldsymbol{x}$, le problème de minimisation précédent est équivalent à maximiser la quantité $h(v)$

$$
h(\boldsymbol{v})=(g \star f)(\boldsymbol{v}) \equiv \iint g(\boldsymbol{x}) f(\boldsymbol{x}-\boldsymbol{v}) \mathrm{d} \boldsymbol{x}
$$

De plus, lorsque le bruit est blanc, l'estimation obtenue est optimale.

\subsection{Approche conventionnelle}

En pratique, deux images sont prises en compte, la première étant l'image de « référence », et la seconde l'image « déformée ». L'image de référence, schématisée sur la figure 3, est quadrillée par un ensemble de ZE qui forme la Région d'Étude (RE). La sous-RE de taille $2^{P} \times 2^{Q}$ pixels est extraite de la $\mathrm{RE}$ de l'image de référence avec les plus grandes valeurs possibles de $P$ et $Q$ pour que celle-ci reste incluse dans la RE. Un premier calcul de corrélation est réalisé par transformée de Fourier rapide sur la sous-RE. La valeur maximale du produit d'intercorrélation permet d'estimer le déplacement moyen $\boldsymbol{v}_{0}$. La RE de l'image déformée est alors centrée en un point correspondant au centre de la RE de l'image de référence déplacé de cette quantité $\boldsymbol{v}_{0}$. Un deuxième calcul est ensuite réalisé sur chaque ZE indépendamment. Sachant qu'un algorithme de transformée de Fourier rapide est utilisé, chaque ZE est un carré de côté $2^{k}$ pixels, où $k$ est un nombre entier. La taille de la ZE est définie par la longueur des côtés. Le maximum de la fonction d'intercorrélation calculée pour chaque ZE donne une première valeur de la correction du déplacement plan $\delta v$. Les centres des ZE dans l'image déformée sont alors décalés de cette quantité $\delta v$. Un algorithme sub-pixel, 


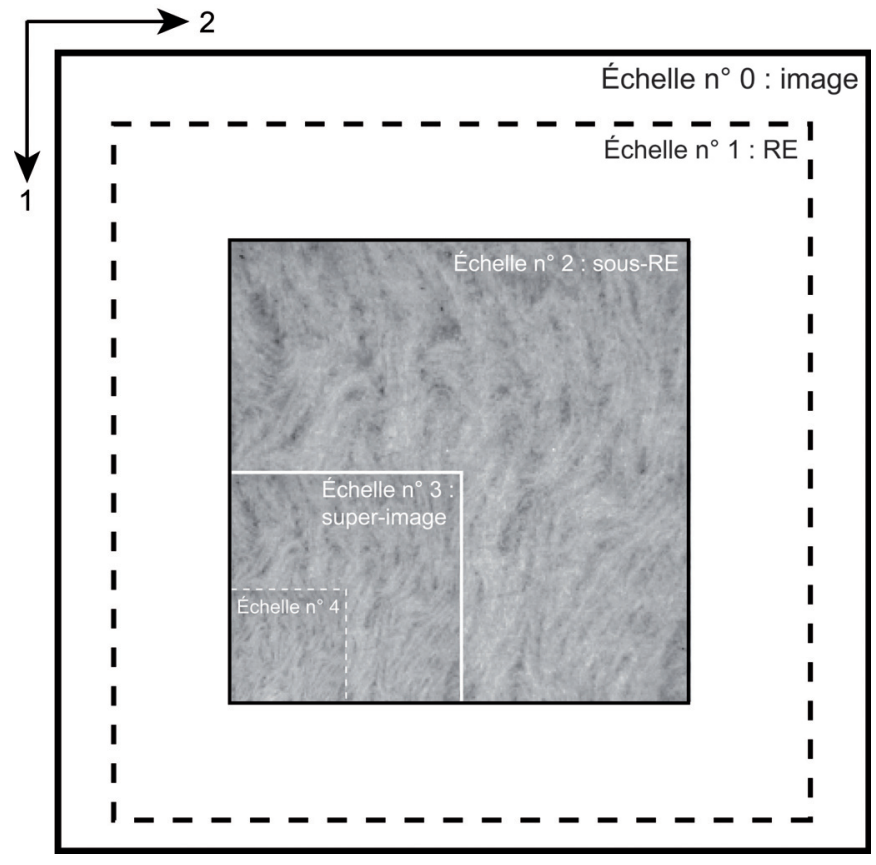

Fig. 4. Différentes échelles utilisées lors de l'approche multiéchelles de corrélation. Dans le cas présent, quatre échelles sont utilisées.

basé sur l'interpolation bi-parabolique de la fonction d'intercorrélation au voisinage de son maximum, permet ensuite d'obtenir des estimations dont la résolution et l'incertitude sont de l'ordre du centième de pixel pour des images codées sur 8 bits [42].

Cette première approche est bien adaptée en petites perturbations, mais si le déplacement est trop important, l'information contenue dans la ZE de l'image de référence ne se trouvera plus que partiellement dans la ZE de l'image déformée, et la correction $\delta v$ ne pourra pas être déterminée de manière fiable. Pour travailler dans le domaine des grandes déformations, comme dans le cas de la laine de verre, une ZE de grande taille serait nécessaire au calcul ce qui a pour inconvénient majeur de moyenner le déplacement sur une surface plus importante. Or le but de la corrélation est de déterminer les champs de déplacement à l'échelle des hétérogénéités de la texture. Pour cela, il faut donc augmenter la déformation maximale mesurable, ce qui est possible grâce à une approche multi-échelles [33].

\subsection{Approche multi-échelles}

Le but est d'améliorer la résolution spatiale (i.e., diminuer la taille des ZE), tout en autorisant un grand déplacement entre deux images consécutives. On applique l'approche précédente de manière récursive sur des images à des échelles différentes. Dans un premier temps, il s'agit d'évaluer des déformations moyennes obtenues à partir de l'analyse avec des images où les détails les plus fins ont été supprimés (i.e., des super-images, comme le montre la Fig. 4). Afin de rendre l'évaluation plus robuste, on applique les déplacements interpolés aux ZE analysées et on ré-évalue ceux-ci jusqu'à ce que la différence maximale entre deux itérations soit inférieure à une limite choisie. À ce moment, on peut rajouter des détails à l'image (i.e., changer d'échelle) et utiliser l'interpolation du déplacement comme première évaluation du champ à cette nouvelle échelle. On procède de la même manière que précédemment jusqu'à revenir à la $\mathrm{RE}$ initiale (avec tous ses détails). Lors de cette dernière étape, le déplacement n'est plus interpolé et on peut ainsi analyser les fluctuations dont les amplitudes sont supérieures à celles accessibles avec l'approche standard.

La figure 4 illustre la construction des images aux différentes échelles. L'échelle $\mathrm{n}^{\circ} 0$ est celle de l'image considérée et l'échelle $n^{\circ} 1$, celle de la RE. L'échelle $n^{\circ} 2$, extraite de la RE, est déterminée par les plus grandes valeurs de $P$ et $Q$ (pour que celle-ci reste incluse dans la $\mathrm{RE}$ ) telles que la taille correspondante de la sous-RE soit $2^{P} \times 2^{Q}$ pixels (Fig. 3 ). À partir de l'échelle $\mathrm{n}^{\circ} 3$, chaque transition d'échelle est caractérisée par la définition de super-pixels : le niveau de gris d'un super-pixel correspond à la moyenne des niveaux de gris des $2 \times 2$ pixels voisins. Cette procédure est poursuivie jusqu'à une taille minimale de la super-image (ici égale à 128 super-pixels). L'analyse commence à l'échelle $\mathrm{n}^{\circ} 4$, par exemple, pour aboutir in fine à l'échelle $\mathrm{n}^{\circ} 1$. De cette manière, un gain d'un facteur quatre peut être attendu sur la déformation maximale mesurable bien que le déplacement maximal mesurable reste limité [33], de la même manière que pour l'approche standard, mais uniquement à l'échelle la plus grossière (ici l'échelle $n^{\circ} 4$ ). Le lecteur intéressé pourra se reporter à la référence [33] pour davantage de détails.

\subsection{Performances de la technique de mesure}

Le but de cette partie est d'évaluer l'incertitude de mesure et la résolution associées à l'algorithme de corrélation présenté ci-dessus. Deux quantités cinématiques sont considérées en fonction de la situation analysée : les déplacements et les déformations. Afin d'avoir des évaluations indépendantes, les paramètres de corrélation sont tels que le décalage $\delta$ est égal à la taille des $\mathrm{ZE}$ (Fig. 3). Il est à noter que les valeurs indiquées ici sont issues d'une analyse sur une image représentative des essais analysés. En effet, chaque texture conduit à des performances différentes en termes de résolution et d'incertitude associées à une résolution spatiale.

Afin d'évaluer l'incertitude moyenne, on impose artificiellement à une image telle que celle montrée en figure 1 des déplacements variant de 0 à 1 pixel par pas de 0,1 pixel. La procédure de déplacement utilise la propriété de décalage/modulation associée à la transformée de Fourier [42]. L'incertitude moyenne correspond à la moyenne des écarts types observés. Pour une taille de ZE de 16 pixels (respectivement 32 pixels, 64 pixels), on obtient une incertitude moyenne inférieure à $2,5 \times 10^{-2}$ pixel (respectivement $1,4 \times 10^{-2}$ pixel, $0,7 \times 10^{-2}$ pixel). Ce résultat montre que plus la taille de la $\mathrm{ZE}$ est grande (i.e., on dégrade la résolution spatiale), plus l'incertitude 
est faible. On montre ainsi qu'il y a un compromis à trouver entre incertitude et résolution spatiale (i.e., taille de la $\mathrm{ZE}$ ).

L'incertitude en termes de deformation est évaluée avec les mêmes images. À partir du champ de déplacement, les déformations sont calculées à l'aide d'un algorithme classique de différences finies centrées de telle manière que la longueur de jauge est égale à $2 \delta$. On définit la résolution spatiale pour la mesure de déformation par cette quantité. Pour une taille de ZE de 16 pixels (respectivement 32 pixels, 64 pixels), on obtient une incertitude moyenne inférieure à $0,13 \%$ (respectivement $0,04 \%, 0,009 \%)$. On observe à nouveau qu'incertitude et résolution spatiale sont liées et que si l'on veut diminuer l'incertitude, on est obligé d'augmenter la taille de la « jauge optique » (i.e., au détriment de la résolution spatiale).

Un bon compromis dans le cas présent est obtenu pour une taille de $\mathrm{ZE}$ de 32 pixels (qui correspond à la résolution spatiale pour les déplacements). En termes de déformation, lorsque le décalage $\delta$ entre ZE est pris égal à 32 pixels (respectivement 16 pixels), la résolution spatiale correspondante vaut 64 pixels (respectivement 32 pixels). Pour évaluer la résolution en déplacement, (i.e., la variation la plus petite de déplacement imposé qui provoque une variation détectable par l'algorithme de corrélation) on procède de la manière suivante. On impose artificiellement un déplacement de la même manière que précédemment. On observe que pour une valeur proche de l'incertitude moyenne, le déplacement moyen peut être détecté de manière raisonnable (i.e., l'erreur relative moyenne entre la valeur imposée et mesurée est inférieure à $8 \%$ ). Pour les déformations, on déforme artificiellement les images par interpolation linéaire des niveaux de gris. Pour une déformation moyenne imposée de $0,01 \%$, on obtient une valeur à $20 \%$ près. La résolution en déformation est ainsi estimée à 0,01\%.

Enfin, des déformations de l'ordre de $\pm 30 \%$ peuvent être mesurées [33,43] entre deux images. Quand une séquence de plus de 2 images est analysée et lorsque l'image de référence est réactualisée (i.e., elle correspond à l'image déformée de l'étape précédente), il n'existe pas de limite à l'approche. Il faut néanmoins remarquer que les erreurs de mesure sont cumulées lors de ce type de traitement. Dans la section suivante, cette technique est appliquée à l'analyse d'essais mécaniques sur laine de verre crêpée.

\section{Essais mécaniques sur laine de verre crêpée}

Afin de déterminer les propriétés mécaniques de la laine de verre crêpée, des essais de compression et d'arrachement sont réalisés. Tous les essais présentés ici sont pilotés en déplacement (ou, de manière équivalente, la déformation globale $\bar{\epsilon}$ est contrôlée; elle est définie comme le rapport entre le déplacement des deux plateaux et la hauteur initiale de l'échantillon). Une compression uniaxiale (dans la direction 1) jusqu'à -20\% de

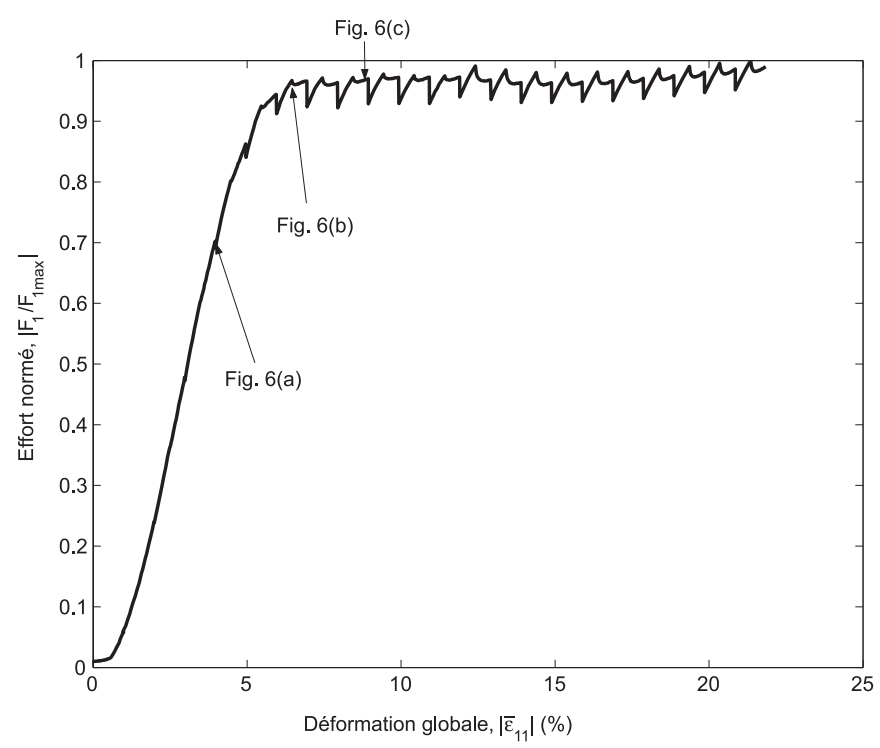

Fig. 5. Effort normé vs. déformation globale pour un essai de compression sur laine de verre crêpée.

déformation globale est appliquée sur des éprouvettes parallélépipédiques de dimension $200 \times 100 \times 80 \mathrm{~mm}^{3}, 80 \mathrm{~mm}$ étant la hauteur de l'éprouvette. Des essais d'arrachement (dans la direction 1) jusqu'à environ $15 \%$ de déformation globale sont également réalisés sur des éprouvettes parallélépipédiques de dimension $200 \times 200 \times 80 \mathrm{~mm}^{3}$ (Fig. 1). Pour ces essais, des images de l'éprouvette éclairée en lumière directe ont été prises pour différents niveaux de déformation globale (i.e., avec des incréments de $-0,5 \%$ en compression et $+0,5 \%$ en arrachement).

\subsection{Essais de compression uniaxiale}

Le résultat d'un essai de compression uniaxiale est présenté sur la figure 5. Le plateau «plastique » suggère l'existence de déformations localisées d'où l'intérêt d'étudier le champ de déformation du milieu avec une bonne résolution spatiale. Sur la figure 6, les champs de déformation sont représentés. Le calcul, réalisé avec des $\mathrm{ZE}$ de taille 32 pixels (i.e., résolution spatiale en déplacement) et avec un décalage de 32 pixels (i.e., la résolution spatiale en déformation est égale à 64 pixels), correspond à la composante $\epsilon_{11}$ du tenseur de déformation (nominale) globale pour différents niveaux de déformation nominale globale, à savoir, $-4 \%$ pour la figure $6 \mathrm{a},-6,5 \%$ pour la figure $6 \mathrm{~b}$ et $-9 \%$ pour la figure $6 \mathrm{c}$, respectivement. Mille cent dix ZE sont considérées par niveau de déformation globale. Sur la figure 5, les points correspondants sont situés à la fin du domaine élastique, au début du plateau «plastique » et là où le plateau « plastique » est bien défini.

Les figures 6 montrent l'évolution du champ de déformation au cours de l'essai : les champs de déformation nominale dans la direction de sollicitation $\epsilon_{11}$ cumulés (depuis le début du test) et les champs incrémentaux qui correspondent à un incrément de 

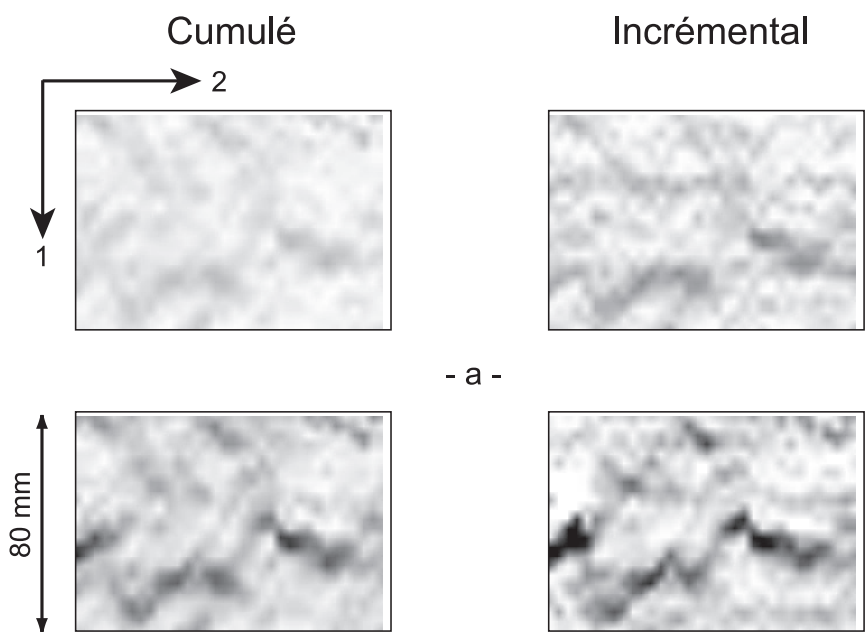

- a -

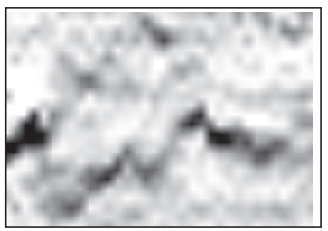

$-b-$
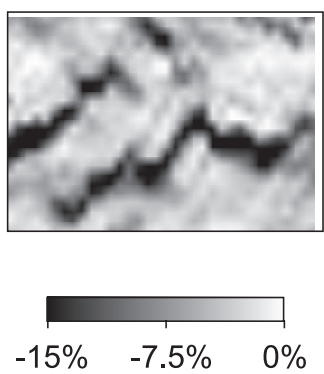

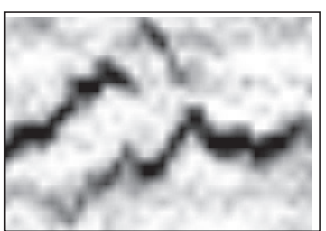

- C -

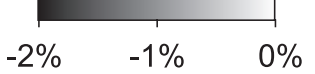

Fig. 6. Champ $\epsilon_{11}$ cumulé et itératif pour différents niveaux de déformation globale : $\bar{\epsilon}_{11}=-4 \%(\mathrm{a}), \bar{\epsilon}_{11}=-6,5 \%$ (b) et $\bar{\epsilon}_{11}=-9 \%$ (c). Paramètres de corrélation : taille de $\mathrm{ZE}=$ 32 pixels, $\delta=32$ pixels ( 1 pixel $\leftrightarrow 0,085 \mathrm{~mm}$ ).

déformation globale de $-0,5 \%$ entre deux images. Dès $-4 \%$ de déformation globale sur la figure $6 \mathrm{a}$, des hétérogénéités de déformation peuvent être mises en évidence à la surface du matériau. Après $-9 \%$ de déformation globale, la zone de localisation bien marquée se dédouble en deux bandes sur la gauche de l'échantillon. Des précurseurs de ces bandes de localisation peuvent déjà être identifiés en amont dans l'essai (clairement sur la Fig. 6b, moins facilement sur la Fig. 6a). Les déformations transverses restent faibles en dehors des bandes de localisation. Leur niveau maximal est du même ordre de grandeur que celui des déformations longitudinales à l'intérieur de ces bandes. Il en est de même pour les déformations de cisaillement dans les zones de localisation non perpendiculaires à la direction de sollicitation.

Tout au long de l'essai, les fluctuations de déformation sont localisées exclusivement dans les bandes précédemment identifiées, et la largeur de ces bandes reste constante. De plus, les niveaux de déformation incrémentale évoluent au sein des bandes de localisation au cours de l'essai. Ces deux phénomènes peuvent être observés sur les cartes de déformation incrémentale, figure 6. À la fin de l'essai, la bande de localisation a une largeur d'environ $8 \mathrm{~mm}$, i.e., un dixième de la hauteur de l'éprouvette. Notons enfin que la résolution et l'incertitude moyenne sont petites par rapport aux niveaux

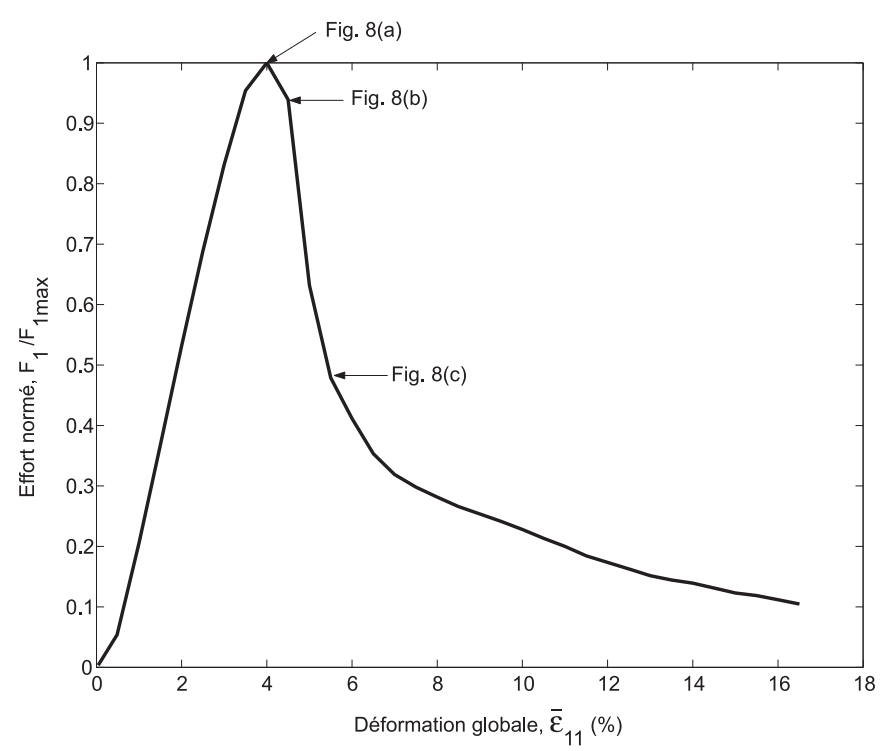

Fig. 7. Effort normé vs. déformation globale pour un essai d'arrachement sur laine de verre crêpée.
Cumulé
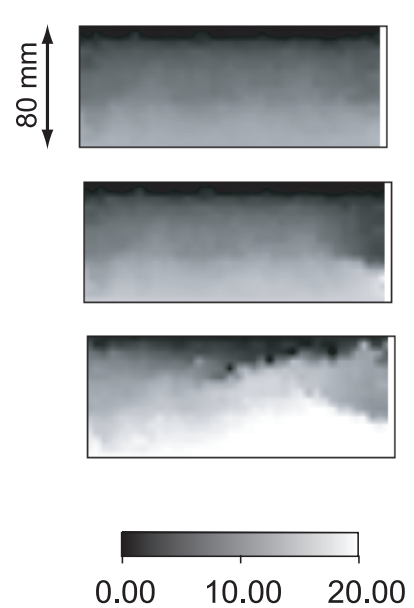

Incrémental

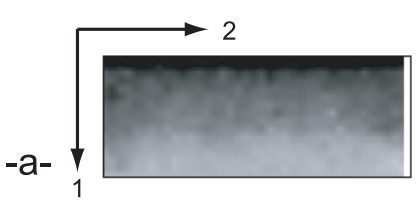

$-b-$
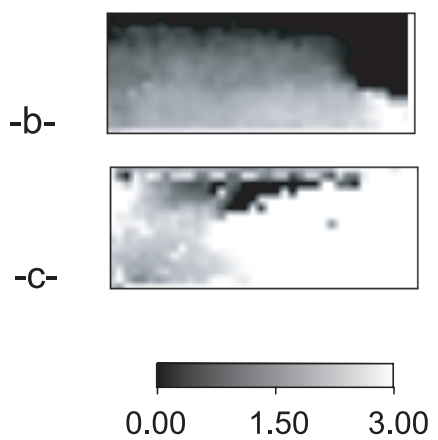

Fig. 8. Champ de déplacement $u_{1}$ cumulé et itératif, en pixels, pour différents niveaux de déformation globale : $\bar{\epsilon}_{11}=$ $4 \%$ (a), $\bar{\epsilon}_{11}=4,5 \%$ (b) et $\bar{\epsilon}_{11}=5,5 \%$ (c). Paramètres de corrélation : taille de $\mathrm{ZE}=32$ pixels, $\delta=32$ pixels $(1$ pixel $\leftrightarrow$ $0,16 \mathrm{~mm})$.

atteints; on peut ainsi conclure que les résultats présentés ici décrivent la réalité expérimentale.

\subsection{Essais d'arrachement}

Le résultat global de l'essai d'arrachement est présenté sur la figure 7. La fin du domaine élastique correspond à l'apparition d'une première fissure. Peu après, une seconde fissure apparaît à la surface de l'éprouvette qui occulte la première et empêche son ouverture. La figure 8 montre les résultats obtenus par corrélation d'images. Le calcul, effectué sur des ZE de taille 32 pixels avec un 
décalage de 32 pixels entre deux ZE consécutives, correspond au déplacement vertical $u_{1}$ pour différents niveaux de déformation globale, à savoir, $4 \%$ pour la figure $8 \mathrm{a}$, $4,5 \%$ pour la figure $8 \mathrm{~b}$ et $5,5 \%$ pour la figure $8 \mathrm{c}$, respectivement. La RE (Fig. 1) contient $624 \mathrm{ZE}$ par niveau de déformation globale.

Sur la figure 8a, le champ de déplacement $u_{1}$ à la surface du matériau correspond à l'apparition de la première fissure. À ce moment, le champ de déplacement devient hétérogène. Sur la figure $8 \mathrm{~b}$, des discontinuités dans les champs de déplacement verticaux $u_{1}$, incrémentaux et cumulés, peuvent être observées. Elles correspondent à l'ouverture d'une fissure. Sur la figure 8c, une autre fissure apparaît à la surface de l'éprouvette. Celle-ci se traduit par une discontinuité des déplacements qui peut être observée sur la carte des déplacements cumulés. Sur la carte des déplacements incrémentaux, on peut observer cette discontinuité, mais aussi l'arrêt de la propagation de la première fissure, écrantée par la seconde. À la fin de l'essai, la fissure traverse complètement l'éprouvette et constitue le mode de rupture final. En ce qui concerne les déformations transverses, avant le pic d'arrachement, leurs niveaux sont de deux ordres de grandeur plus faibles que les déformations longitudinales.

Ce calcul de corrélation permet de révéler les discontinuités de déplacement qui correspondent à des fissures à la surface de l'éprouvette. Étant donné qu'il s'agit d'une mesure en surface, on ne peut pas exclure le fait que les fissures puissent s'amorcer préalablement au sein du matériau, sans atteindre la face analysée du matériau. Enfin, à l'instar de l'essai de compression, les valeurs de résolution en déplacement et l'incertitude moyenne correspondante sont faibles par rapport aux niveaux mesurés; ceci permet d'avoir une certaine confiance dans les résultats obtenus, qui sont également corroborés par des observations visuelles post-mortem.

\subsection{Influence des paramètres de corrélation}

Des calculs ont également été réalisés en utilisant d'autres paramètres de corrélation (e.g., un décalage $\delta$ entre deux ZE consécutives plus petit) pour étudier leur influence sur les résultats. Les deux essais sont à nouveau analysés.

Pour l'essai de compression, on s'attend, par exemple, à ce que la largeur de la bande de localisation dépende du décalage entre deux $\mathrm{ZE}$ consécutives. Un calcul avec un décalage $\delta$ de 16 pixels a été effectué (i.e., la résolution spatiale en déformation est égale à 32 pixels). La figure 9 montre les résultats de corrélation pour les mêmes niveaux de déformation que les résultats présentés à la section 4.1 avec un décalage de 32 pixels. Lors d'un tel calcul, pour la même RE, le nombre de ZE est quatre fois plus élevé qu'avec un décalage de 32 pixels. Si l'on compare les résultats, on peut observer que la localisation de la déformation apparaît pour la même image et que les deux bandes identifiées sont situées au même endroit. De plus, sur les figures 9 et 6 , la largeur des bandes

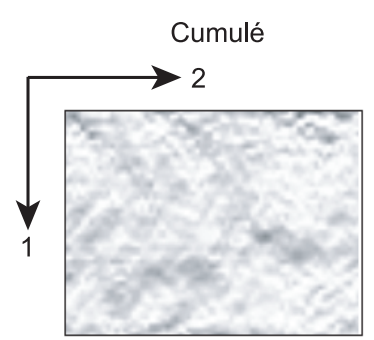

Incrémental

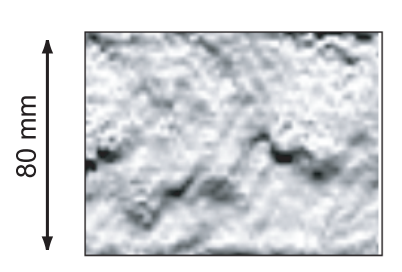

-a-
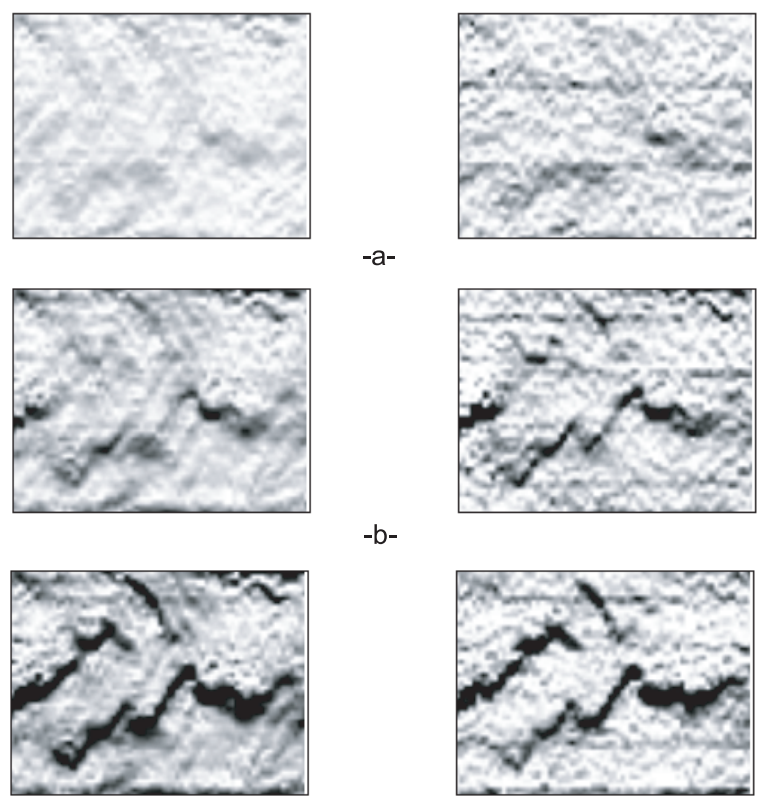

$-b-$
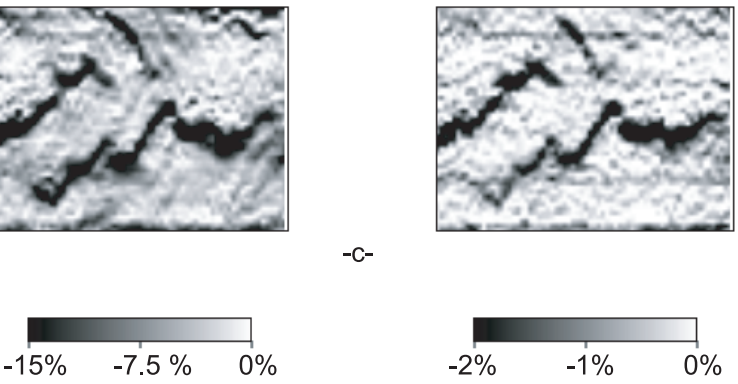

$-\mathrm{C}-$

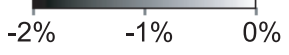

Fig. 9. Champ $\epsilon_{11}$ cumulé et itératif pour différents niveaux de déformation globale : $\bar{\epsilon}_{11}=-4 \%$ (a), $\bar{\epsilon}_{11}=-6,5 \%$ (b) et $\bar{\epsilon}_{11}=-9 \%$ (c). Paramètres de corrélation : taille de $\mathrm{ZE}=$ 32 pixels, $\delta=16$ pixels ( 1 pixel $\leftrightarrow 0,085 \mathrm{~mm}$ ).

reste la même alors que le nombre de ZE composant cette bande est différent. Pour que les niveaux de déformation puissent être comparés, les deux calculs ont la même échelle d'analyse. Dans le cas présent, l'influence des paramètres de corrélation est faible tant que l'échelle de mesure reste adaptée au mécanisme que l'on souhaite mettre en évidence (e.g., la localisation de la déformation).

Pour les essais d'arrachement, la différence entre les deux calculs pour des décalages $\delta$ différents est plus importante car une fissure correspond à une discontinuité dans le champ de déplacement. Si la fissure est contenue dans seulement une ZE, la discontinuité représentera la fissure entière, mais si la largeur de la fissure comprend plusieurs ZE, les lèvres de la fissure peuvent être visibles. La figure 10 montre les résultats de corrélation pour un décalage $\delta$ de 16 pixels. Avec ce dernier, pour la même RE, le nombre de ZE est quatre fois plus élevé qu'avec un décalage de 32 pixels. Les résultats pour les mêmes niveaux de déformation globale que ceux présentés à la section 4.2 sont présentés sur la figure 8 . Sur le champ incrémental, on peut observer, pour un décalage de 16 pixels, que le champ de déplacement est plus hétérogène à la surface du matériau. Pour 4,5\% de déformation globale (Fig. 10b), la première fissure peut facilement être identifiée. Les lèvres de la fissure apparaissent à la surface du matériau, ce qui n'était pas visible pour un décalage de 32 pixels. Pour ce dernier calcul, 
Cumulé
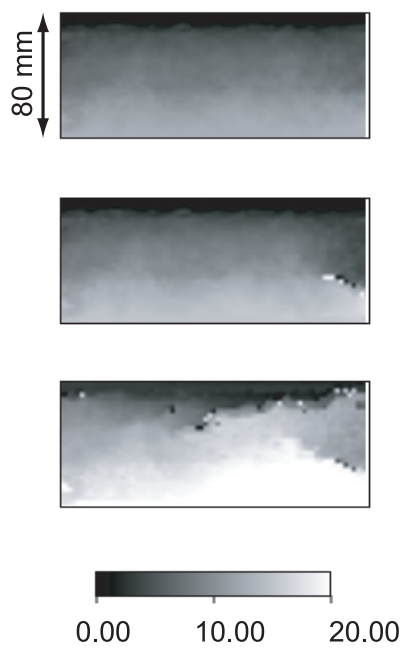

Incrémental

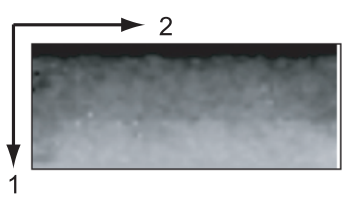

-a-

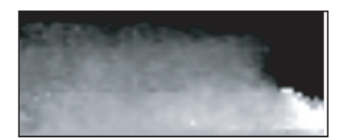

$-b-$

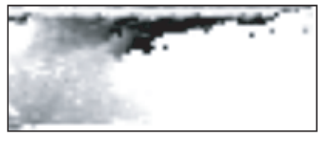

$-c-$

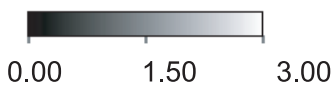

Fig. 10. Champ de déplacement $u_{1}$ cumulé et itératif, en pixels, pour différents niveaux de déformation globale : $\bar{\epsilon}_{11}=$ $4 \%$ pour (a), $\bar{\epsilon}_{11}=4,5 \%$ pour (b) et $\bar{\epsilon}_{11}=5,5 \%$ pour (c). Paramètre de corrélation : taille de la $\mathrm{ZE}=32$ pixels, $\delta=16$ pixels $(1$ pixel $\leftrightarrow 0,16 \mathrm{~mm})$.

la fissure est formée de plusieurs ZE et son ouverture est visible. Sur la figure 10c, la deuxième fissure est également visible. Les résultats du calcul de corrélation dépendent donc des paramètres utilisés. Leur influence est nette sur les résultats, de manière contrôlée toutefois. Si la méthode est utilisée pour détecter la présence de fissures, un calcul plus grossier, avec un décalage entre deux ZE de même valeur que la taille d'une ZE est suffisamment efficace. Si l'on recherche la taille de la fissure, un calcul plus fin est nécessaire.

\section{Conclusion}

Une nouvelle approche de la mesure de champs de déplacement et de déformation a été présentée. Au-delà de la technique conventionnelle de corrélation d'images numériques, qui permet une première évaluation du champ de déplacement, l'image est redimensionnée et repositionnée à une échelle réduite pour augmenter à la fois la robustesse de la technique et la déformation maximale mesurable entre deux images consécutives. Cette approche multi-échelles a été utilisée pour analyser des essais mécaniques en réactualisant l'image de référence.

Cette technique a tout d'abord été appliquée à un test de compression uniaxiale sur une éprouvette en laine de verre crêpée. Elle a permis d'identifier des zones d'hétérogénéités de la déformation ainsi que les bandes de localisation de la déformation. Si les paramètres du calcul de corrélation sont modifiés, les résultats restent très proches, c'est-à-dire que les niveaux de déformation sont les mêmes, les hétérogénéités apparaissent pour le même niveau de déformation globale, et les bandes de localisation ont la même largeur pour un niveau de déformation donné (très supérieur à la résolution et à l'incertitude moyenne de mesure).

En utilisant la même procédure, des essais d'arrachement ont été réalisés sur laine de verre crêpée. Des discontinuités dans le champ de déplacement, prémices de l'arrachement ou de l'apparition de fissure, peuvent être observées. L'influence des paramètres de calcul a été quantifiée. Si seule la présence d'une fissure ou d'un champ de localisation est recherchée, un calcul rapide et approximatif est suffisant, tandis que si une analyse détaillée est demandée, une échelle plus fine est nécessaire. Les niveaux de déplacements mesurés sont très supérieurs à la résolution et à l'incertitude de mesure par corrélation d'images.

La technique de corrélation appliquée à la laine de verre crêpée montre le changement de macrostructure au cours des essais. La laine de verre étant un matériau fortement texturé, les orientations locales des fibres constitutives ont également été caractérisées afin d'étudier le lien entre cette texture initiale et la zone de localisation de la déformation ou de fissuration. Un développement à venir est de relier les champs de déplacement et de déformation obtenus aux hétérogénéités locales de la texture de la laine de verre crêpée et d'évaluer les performances mécaniques de la texture, afin d'optimiser l'opération de crêpage.

Remerciements. Ce travail est soutenu par Isover SaintGobain et par le CNRS dans le cadre d'un projet intitulé « Analyses multi-échelles de champs de déformation par traitement d'image : vers l'identification de champs de propriétés mécaniques ». Les auteurs remercient également O. Gaume, C. Machelart et J.-B. Rieunier pour d'utiles discussions.

\section{Références}

[1] T.F. Drouillard, A History of Acoustic Emission. J. Acoust. Em. 14(1) (1996) 1-34

[2] ISO 3057-1974, NDT-Metallographic Replica Techniques of Surface Examinations, 1974

[3] P.K. Rastogi (éd.) Photomechanics, Springer, Berlin, 2000

[4] P. Jacquot, J.-M. Fournier (éd.) Interferometry in Speckle Light, Theory and Applications, Springer, Berlin, 2000

[5] D.S. Dawicke, M.S. Sutton, CTOA and Crack-Tunneling Measurements in Thin Sheet 2024-T3 Aluminum Alloy, Exp. Mech. 34 (1994) 357-368

[6] L. Humbert, V. Valle, M. Cottron, Experimental Determination and Empirical Representation of Out-ofPlane Displacements in a Cracked Elastic Plate Loaded in Mode I, Int. J. Solids Struct. 37 (2000) 5493-5504

[7] J. Desrues, J. Lanier, P. Stutz, Localization of the Deformation in Tests on Sand Samples, Eng. Fract. Mech. 21 (1985) 251-262

[8] Y. Berthaud, D. Paraskevas, M. Taroni (éd.) Photomécanique 95 (GAMAC, Paris), 1995

[9] E.G. Coker, L.N.G. Filon, A Treatise on Photoelasticity, Cambridge University Press, New York, 1931

[10] M.M. Frocht, Photoelasticity, volume 1, John Wiley and Sons, New York, 1941 
[11] M.M. Frocht, Photoelasticity, volume 2, John Wiley and Sons, New York, 1948

[12] H.T. Jessop, F.C. Harris, Photoelasticity : Principles and Methods, Dover, New York, 1949

[13] J. Guild, Diffraction Gratings as Measuring Scales, Practical Guide to Metrological Use of Moiré Fringes, Oxford University Press, Oxford, 1960

[14] P.S. Theocaris, Moiré Fringes in Strain Analysis, Pergamon Press, London, 1969

[15] A.J. Durelli, V.J. Parks, Moiré Analysis of Strain, Prentice-Hall, Englewood Cliffs, 1969

[16] R.L. Powell, K.A. Stetson, Interferometric Vibration Analysis by Wavefront Reconstruction, J. Opt. Soc. Am. 55 (1965) 1593-1598

[17] K.A. Stetson, R.L. Powell, Interferometric Hologram Evaluation and Real-Time Vibration Analysis of Diffuse Objects, J. Opt. Soc. Am. 55 (1965) 1694-1695

[18] P. Smigielski, Holographie industrielle, Teknea, Toulouse, 1994

[19] J.M. Burch, Interferometry with Scattered Light, in J.H. Dickson, (éd.), Optical Instruments and Techniques, Oriel Press, London, 1970, pp. 213-229

[20] J.A. Leendertz, Interferometric Displacement on Scattering Surfaces Utilizing Speckle Effect, J. Phys. E, Sci. Instrum. 3 (1970) 214-218

[21] E. Archbold, A.E. Ennos, P.A. Taylor, A Laser Speckle Interferometer for the Detection of Surface Movements and Vibration, in Dickson, J.H. (éd.), Optical Instruments and Techniques, Oriel Press, London, 1970, pp. $265-275$

[22] T.D. Dudderar, P.G. Simpkins, Laser Speckle Photography in a Fluid Medium, Nature 270 (1977) $45-47$

[23] C.J.D. Pickering, N.A. Halliwell,, Speckle Laser in Fluid Flows : Signal Recovery with Two-Step Processing, Appl. Opt. 23 (1984) 1128-1129

[24] W. Merzkirch, Flow Visualisation, Academic, New York, USA, 1987

[25] P.J. Burt, C. Yen, X. Xu, Local Correlation Measures for Motion Analysis : a Comparative Study, in Proceedings IEEE Conf. on Pattern Recognition and Image Processing, 1982, pp. 269-274

[26] W.H. Peters, W.F. Ranson, Digital Imaging Techniques in Experimental Stress Analysis, Opt. Eng. 21 (1982) 427-431

[27] M.A. Sutton, W.J. Wolters, W.H. Peters, W.F. Ranson, S.R. McNeill, Determination of Displacements Using an Improved Digital Correlation Method, Im. Vis. Comp. 1 (1983) 133-139

[28] R.J. Adrian, Particle Imaging Techniques for Experimental Fluid Mechanics, Ann. Rev. Fluid Mech. 23 (1991) 261-304

[29] M. Raffel, C.E. Willert, J. Kompenhans, Particle Image Velocimetry, a Practical Guide, Springer, Berlin Germany, 1998
[30] C.E. Willert, M. Gharib, Digital Particle Image Velocimetry, Exps. Fluids 10 (1991) 181-193

[31] D. Garcia, J.-J. Orteu, Mesure de formes et de déformations 3D par stéréo-corrélation : application sur des tôles minces embouties, in Proceedings Nouveaux moyens optiques pour l'industrie, SFO, club Contrôles et Mesures Optiques pour l'Industrie, 1999, pp. 177-184

[32] M.A. Sutton, S.R. McNeill, J.D. Helm, Y.J. Chao, Advances in Two-Dimensional and Three-Dimensional Computer Vision in Photomechanics, Springer, Berlin, Germany, 2000, pp. 323-372

[33] F. Hild, B. Raka, M. Baudequin, S. Roux, F. Cantelaube, Multi-Scale Displacement Field Measurements of Compressed Mineral Wool Samples by Digital Image Correlation, Appl. Optics 41 (2002) 6815-6828

[34] C. G'Sell, J.-M. Hiver, A. Dahnoun, A. Souahi, VideoControlled Tensile Testing of Polymers and Metals Beyond the Necking Point, J. Mat. Sci. 27 (1992) 5031-5039

[35] L. Chevalier, S. Calloch, F. Hild, Y. Marco, Digital Image Correlation used to Analyze the Multiaxial Behavior of Rubber-Like Materials, Eur. J. Mech. A/Solids 20 (2001) 169-187

[36] S. Roux, F. Hild, Y. Berthaud, Correlation Image Velocimetry : A Spectral Approach, Appl. Optics 41 (2002) 108-115

[37] D. Choi, J.L. Thorpe, R. Hanna, Image Analysis to Measure Strain in Wood and Paper, Wood Sci. Technol. 25 (1991) 251-262

[38] T.C. Chu, W.F. Ranson, M.A. Sutton, W.H. Petters,, Applications of Digital-Image-Correlation Techniques to Experimental Mechanics, Exp. Mech. 3 (1985) 232-244

[39] D.J. Chen, F.P. Chiang, Y.S. Tan, H.S. Don, Digital Speckle-Displacement Measurement Using a Complex Spectrum Method, Appl. Opt. 32 (1993) 1839-1849

[40] Y. Berthaud, J. Scholz, J. Thesing, Méthodes optiques et acoustiques de mesures des caractéristiques mécaniques, Proceedings Colloque national MECAMAT « Mécanismes et mécanique des grandes déformations », 1996, pp. $77-80$

[41] F.P. Chiang, Q. Wang, F. Lehman, New Developments in Full-Field Strain Measurements Using Speckles. In Non-Traditional Methods of Sensing Stress, Strain and Damage in Materials and Structures, ASTM, Philadelphia USA, 1997, pp. 156-169

[42] J.N. Périé, S. Calloch, C. Cluzel, F. Hild, Analysis of a Multiaxial Test on a C/C Composite by Using Digital Image Correlation and a Damage Model, Exp. Mech. 42 (2002) 318-328

[43] L. Chevalier, Y. Marco, Tools for Multiaxial Validation of Behavior Laws Chosen for Modeling Hyper-Elasticity of Rubber-Like Materials, Polym. Eng. Sci. 42 (2002) 280-298 